\title{
LA ESCUELA DE ÁMSTERDAM, LA BELLEZA COMO IDEARIO SOCIAL ${ }^{1}$
}

\author{
Noud de Vreeze \\ Doctor Ingeniero
}

\section{André Ouwehand}

Ingeniero, Senior researcher urban renewal and housing

OTB, the Research Institute

Palabras Clave: escuela de arquitectura de Ámsterdam, Tellegen, Wibaut, Keppler, Berlage y De Klerk

Resumen: Entre los años 1920 y 1940 particulares y corporaciones de vivienda construyeron en Ámsterdam más de 80.000 viviendas, que se sumaron a las aproximadamente 140.000 viviendas existentes. Entre los proyectos más llamativos de este período están los complejos residenciales diseñados por arquitectos de la llamada "Escuela de Ámsterdam", cuyo representante más significativo fue Michiel de Klerk. Los ejemplos más hermosos no son villas aisladas destinadas a las clases más pudientes, sino viviendas sociales de alquiler construidas por encargo de las corporaciones de vivienda. Los llamaban "Palacios para los trabajadores": la belleza como ideario social. Fueron construidas dentro del marco de nueva legislación gracias a la colaboración de políticos, arquitectos-urbanistas, corporaciones de vivienda y arquitectos. Muestras de una época que se caracterizó por el compromiso social, optimismo, dinamismo e idealismo.

En este artículo se tratan estos pilares con el orden siguiente: la Ley de la Vivienda de 1901, el surgimiento de las corporaciones de vivienda, los cambios en la política municipal y la trama tan especial que surgió a principios del siglo XX. Esto dio lugar a las hermosas manzanas de los arquitectos de la Escuela de Ámsterdam dentro de los nuevos ensanches de la ciudad. En los últimos años se han desarrollado muchos esfuerzos para conservar adecuadamente este legado arquitectónico y en algunas ocasiones para devolverlo a su honra originaria.

\section{La Ley de la Vivienda (Woningwet) de 1901 como estímulo a la vivienda y el urbanismo}

La situación del alojamiento de los trabajadores en las ciudades neerlandesas cobra actualidad política durante la segunda mitad del siglo XIX. Epidemias de cólera en los años treinta y cuarenta y amenazas continuas a la salud pública provocaron que el rey Willem II ordenase estudios sobre la situación de las viviendas de los trabajadores (Ottens, 1975 p. 8). Ello dio lugar a iniciativas filantrópicas en diferentes ciudades. La construcción seguía en manos del sector privado, y hasta finales del siglo XIX no se produjeron cambios a este sentido. Entonces, la mejora de las condiciones de alojamiento adquiere mucha atención dentro de las cuestiones sociales (Van der Schaar 1987, p. 70- 81). El alegato a favor de mejores viviendas fue apoyado entre otros por el Partido Liberal (Liberale Partij), el socialista Sindicato General de Trabajadores Neerlandeses (Algemeen Nederlandse Werklieden Verbond), y Patrimonium, el sindicato de trabajadores cristiano-protestantes. Los sindicatos de trabajadores no eran tanto organizaciones revolucionarias, sino que perseguían más bien ennoblecer a la clase trabajadora dentro de las condiciones sociales de la época. Ya antes de la aprobación de la Ley de la Vivienda en 1901 desplegaron pequeñas iniciativas para la construcción de viviendas.

\footnotetext{
${ }^{1}$ Demetrio Muñoz Gielen, Traducción y notas de investigador en el Instituto de Investigación OTB
} 
Finalmente, este interés político por la materia lleva a la Ley de la Vivienda de 1901. Para poner fin a las malas condiciones de alojamiento se introdujeron cuatro importantes medidas que estaban relacionadas entre sí.

En primer lugar la introducción de la obligatoriedad del permiso de construcción que garantizase que la vivienda nueva cumpliese con unos requisitos mínimos.

Una segunda importante condición era la creación de un marco institucional para la construcción de vivienda social y para hacer posible la financiación (por parte del gobierno central) en condiciones óptimas (interés bajo y subsidios) de las asociaciones, fundaciones o sociedades que se dedican exclusivamente a la mejora de las condiciones de alojamientos En tercer lugar la obligación de que en lo sucesivo, los municipios de más de 10.000 habitantes aprobasen un plan general de ensanche, plan que debía ser examinado por los gobiernos provinciales y por funcionarios de la Administración Central. De esta forma las cuestiones relacionadas con el diseño de manzanas, barrios, espacio público y sistemas locales y generales pasaron a formar parte de los procesos de estudio y toma de decisiones dentro de la administración y la política locales.

En cuarto lugar se introdujo la obligación de que una comisión local examinase y aprobase los aspectos estético-arquitectónico de cada iniciativa de construcción antes de otorgar el permiso.

\section{Corporaciones de vivienda independientes y construcción municipal de viviendas}

La Ley de la Vivienda ofreció la oportunidad a numerosas iniciativas de fundar asociaciones de construcción de viviendas, fundaciones de vivienda o cooperativas de vivienda. Esta posibilidad ofrecida por la ley fue utilizada profusamente. El sector neerlandés de la vivienda social de alquiler se distancia a este sentido, de buena parte de los países de la Europa Occidental, donde la construcción de vivienda social es (ha sido) fundamentalmente una labor de la Administración Municipal (Ouwehand \& Van Daalen, 2002 p. 4). Las organizaciones que contribuyeron a la llegada de la Ley de la Vivienda hicieron profusamente uso de las nuevas posibilidades. Sin embargo, hubo que esperar hasta 1915 para que las posibilidades que la Ley de la Vivienda ofrecía fueran desarrolladas efectivamente por la Administración Central. A partir de este momento el Gobierno central pone a disposición aportaciones substanciales para la construcción de vivienda social. Para entonces en Ámsterdam habían ya tenido lugar cambios políticos de importancia, entre otros los que llevaron a la fundación de la Empresa Municipal de la Vivienda (Gemeentelijke Woningdienst) y de la empresa municipal del suelo, encargada de hacer posible la ejecución de los planes de ensanche por medio de una política activa de suelo (Slot, 1996) ${ }^{2}$.

Todo este sistema sirvió en los años 1920 y 1930 de base para el fuerte desarrollo de una política profesional con connotaciones sociales dentro de estas organizaciones, organizaciones que se necesitaban y aprendían las unas a/de las otras: departamentos municipales para el control de la construcción y la vivienda (bouw- en woningtoezicht), para el desarrollo urbanístico, corporaciones de vivienda, sus dirigentes y organizaciones nacionales, arquitectos y arquitectos-urbanistas.

Tras la llegada de la Ley de la Vivienda, las constructoras privadas eran aún activas en la construcción de viviendas, pero tras el inicio de la Primera Guerra Mundial ${ }^{3}$ la escasez de materiales y capital, que dieron lugar a una rápida subida de los costes de construcción y del precio del dinero, provocaron su casi total retirada. Cuando durante la segunda mitad de los años 1920 las constructoras privadas empezaron a ganar poco a poco terreno gracias a que

\footnotetext{
${ }^{2}$ Nota del traductor: en los Países Bajos políticas activas de suelo han tenido y tienen aún, aunque en menor medida, un gran arraigo en el Urbanismo local. Consisten en que el Ayuntamiento compra o expropia todo o parte del suelo a urbanizar, lo urbaniza y finalmente vende los solares edificables a los promotores de vivienda.

${ }^{3}$ Nota del traductor: durante la Primera Guerra Mundial los Países Bajos mantuvieron una posición neutral.
} 
los subsidios y los préstamos baratos a las corporaciones de viviendas fueron limitadas, en Ámsterdam ya se habían consolidado fuertes tradiciones urbanísticas y arquitectónicas. Presumiblemente la gran continuidad dentro del aparato administrativo del Ayuntamiento hizo posible que una serie de principios arquitectónicos y urbanísticos muy valorados y que un rol dominante de la Administración Local en la urbanización de grandes proyectos urbanísticos se mantuviesen a pesar del retorno de las constructoras privadas.

Además, la Comisión Estético-arquitectónica de Ámsterdam, que examina todas las solicitudes de permiso de construcción y que desempeña un papel influyente, tenía una clara preferencia por el estilo arquitectónico de los arquitectos ligados a la Escuela de Ámsterdam.

\section{La red: Tellegen, Wibaut, Keppler, Berlage y De Klerk}

Dentro del marco creado por la Ley de la Vivienda se pudieron realizar iniciativas de desarrollo urbanístico dirigidos y organizados centralizadamente por el municipio. Muchos ayuntamientos holandeses encargaron la elaboración de grandes planes de ensanche, gracias a los cuales durante un largo período la extensión de las ciudades siguió una estructura 'regular' (o sea, siguiendo unas reglas determinadas).

La política de vivienda social y los ensanches se convirtieron en temas cruciales dentro de la política local. En los decenios posteriores a de 1900 tuvo lugar una revolución silenciosa dentro de la política local amsterdamesa. La élite política liberal fue desplazada lenta pero irrevocablemente por diferentes partidos socialistas que querían utilizar el aparato de la Administración para potenciar programas sociales en los terrenos de la educación, la vivienda, la higiene y la salud pública. Los socialistas lograron en 1903 por primera vez uno de los 45 concejales del Pleno Municipal; en 1919 eran ya veinte.

El éxito de la expansión urbanística amsterdamesa durante el período de entreguerras, entre los años 1920 y 1940, se debe sin duda al contexto creado por la nueva legislación y por el renovado equilibrio político. Pero dentro de este contexto, el éxito se puede también atribuir a una coalición de unos cuantos prominente correligionarios de la política amsterdamesa.

Sabemos que J.W.C. Tellegen, con anterioridad a su período como alcalde (fue alcalde de Ámsterdam de 1915 a 1921), dirigió desde 1901 el departamento municipal de Control de la Construcción y la Vivienda (Bouw- en Woningtoezicht). Tellegen fue el redactor de las primeras Ordenanzas de Edificación (Bouwverordening) de Ámsterdam con base legal, ordenanzas que fueron aplicadas por un nuevo departamento municipal de carácter profesional con supervisores e inspectores (ver Ottens, E., 1975). Tellegen no sólo valoraba las cuestiones técnico-constructivas, sino que valoraba igualmente la calidad arquitectónica.

En una exposición en el Museo Stedelijk organizada en 1915 por la Sociedad Amsterdamesa Architectura et Amicitia, que celebraba así su doceavo lustro, se expusieron sorprendentes trabajos de diseñadores jóvenes que más adelante serían conocidos como los arquitectos de la Escuela de Ámsterdam. Tellegen defendió en su discurso inaugural de una forma explícita la conveniencia de que el diseño de las viviendas se confiase a arquitectos. Él contemplaba como su misión personal “...ayudar a potenciar la hermosa profesión del arquitecto.” (Bock, M., 1997).

Durante el período en que Tellegen fue alcalde, Wibout era el concejal de vivienda social. Tuvo que existir una trama muy estrecha formada por personas e instituciones que trabajaron conjuntamente en un inspirador contexto de nueva legislación y optimismo político en torno a la enorme tarea de construcción. El talentoso, prestigioso y enérgico Wibout entró en 1907 en el Pleno Municipal por el Partido Socialdemócrata de los Trabajadores (Sociaaldemocratische Arbeiderspartij, SDAP), e inició en 1914, cuando contaba ya con 55 años, su brillante carrera como concejal con concejalía en Ámsterdam. Primero la concejalía Vivienda Social, y desde 1919 hasta 1921 además de Vivienda Social, también Hacienda. Después de 1921 un correligionario de partido asumió la concejalía de Vivienda Social. Él permaneció como concejal 
de Hacienda hasta 1931, cuatro años antes de su muerte a los 75 años. Durante su época de concejal introdujo con fuerza el tema de la vivienda social en los debates plenarios. Como miembro del equipo de gobierno municipal imprimió gran influencia en la política local. En 1915, un año después de entrar en el Pleno Municipal, se fundó el Departamento Municipal de la Vivienda (Gemeentelijke Woningdienst). Como miembro del equipo de gobierno municipal dio cobertura política y financiera para poder ejecutar una política de construcción de viviendas dinámica.

Junto a Wibout desempeñó su labor Arie Keppler, personaje conocido como pelmazo y luchador irascible. Fue el director del Departamento de la Vivienda desde 1915 hasta 1937 y, aunque era mucho más joven que Wibout, era su cuñado.

Keppler era una figura muy comprometida y polifacética dentro del sector de la vivienda social. Se esforzó para la realización de 'escuelas vivienda' destinadas a familias inadaptadas socialmente que aprendían allí lo relacionado con la vivienda. Estuvo entre los iniciadores de la construcción del experimental "Pueblo Hormigón" (Betondorp) en Watergraafsmeer, al sudeste de Ámsterdam. Keppler escribió junto con Berlage, Kromhout y Jan Wils, arquitectos y arquitectos-urbanistas con una reputación nacional e internacional, el famoso libro "Viviendas de Trabajadores en los Países Bajos" (Arbeiderswoningen in Nederland), publicado en 1921. Era una defensa de una arquitectura y un urbanismo de gran calidad, justamente al servicio de una política de construcción de viviendas sociales y en defensa de una amplia financiación y subsidios de viviendas para los trabajadores por parte del gobierno central. Este libro se publicó en el momento en que justamente el gobierno central cortó radicalmente con esta política de apoyo a la vivienda social (De Vreeze, 1992, p. 180). Keppler tenía una gran motivación social, e hizo posible que hijos de arrendatarios de viviendas municipales pudiesen ir de vacaciones en campamentos (Ottens, 1975).

Pero Keppler era sobre todo conocido como influyente defensor del trabajo de la Escuela de Ámsterdam. La abundancia lírica de la arquitectura de De Klerk, Kramer, Wijdeveld y Van der Mey tenían en la época la fama de excesos extravagantes. Pero gracias al entusiasmado liderazgo de Keppler y otros defensores de la causa de la vivienda social la calidad de sus diseños se mantuvo como norma durante años. Según Wibaut (crónica en el diario El Pueblo Het Volk-, publicada en 1930, cuando Keppler era ya 15 años director del Departamento de la Vivienda), Keppler había logrado fundamentalmente dos conceptos. Construyó viviendas unifamiliares para trabajadores y vinculó a los arquitectos en la construcción de viviendas. "A veces no sabía si aún se atrevía; pero después de alguna duda decía: 'la belleza es una alegría para siglos'. Él continuó, (y) nos enseñó que la construcción de viviendas ha de ser siempre una mejora, tanto en contenido como en valor arquitectónico". Wibaut se refería aquí a un momento en que Keppler entró en su despacho con la pregunta si la construcción de un edificio de viviendas para trabajadores podía continuar si el coste fuese el doble de lo presupuestado. Wibaut decidió continuar, incluso estaba claro que los alquileres pudiesen mantenerse. "Pero lo que sé con seguridad es que si ahora construimos de inmediato un par de miles de buenas viviendas, éstas servirán para el bienestar de la población, (y) eso ya no se nos podrá arrebatar. Este importante comienzo, que tan amargamente necesario es para la mejora de las condiciones de alojamiento del pueblo, ya será realidad" (Smit, F., 2001).

Todas las fuentes que nos quedan señalan que Tellegen, Wibaut y Keppler tenían un vínculo personal e ideológico muy especial, ellos tenían una misma causa y trabajaban para ella desde el municipio amsterdamés, cada uno desde su propio legado personal y desde sus distintas posiciones. Jugaron un papel en la avanzadilla local y nacional de socialdemócratas y del movimiento de liberales progresistas y tuvieron un gran compromiso personal con e influencia en el mundo de los reformadores de la política de vivienda y las recién fundadas corporaciones de vivienda. Allí, en los círculos de las corporaciones, los líderes del movimiento obrero, la élite intelectual ilustrada y prominentes políticos locales encontraron los medios para desempeñar el rol de señores de la construcción con el apoyo financiero de la Administración Pública. Keppler 
y Wibaut encontraban placer en ello. En el libro conmemorativo de una corporación de viviendas amsterdamesa de la época se describe cómo en el domicilio de Keppler se jugaba los domingos por la mañana a "corporacioncita de vivienda" (woningbouwverenigingetje) (Richter Roegholt, 1993). Él ayudó a menudo a jóvenes dirigentes inexpertos en los vericuetos del mundo oficial de la burocracia pública y la legislación.

\section{Berlage: planes de ensanche como base robusta}

Queda claro que en este clima de ambiciones políticas y de funcionarios públicos se hizo espacio para talentosos y ambiciosos diseñadores. A Berlage se le reconoce ampliamente como la figura central, el 'Grand Old Man' de esta época de renovación.

En 1917, cuando su nueva versión del Plan Sur (Plan-Zuid) fue finalmente aprobado por el Pleno Municipal ${ }^{4}$, Berlage ya tenía 60 años. Como arquitecto sentó las bases para un funcionalismo moderno y racional: defendió como principio-guía para la arquitectura una fuerza expresiva de materiales y construcción y un significado subordinado de los ornamentos y adornos. Su famoso Plan Sur sirvió hasta los años 1930 como el marco para la construcción de 10.000 viviendas. Anchas avenidas situadas de este a oeste forman la estructura principal para una red jerárquica de calles y barrios sutilmente construída, donde la manzana cerrada formaba, por así decirlo, el auténtico ladrillo con el que toda la construcción se montó (Gaillard, 1992).

La estructura principal de avenidas y barrios se prestaba también para posicionar estratégicamente una serie de edificios monumentales de importancia que tenían una función pública, como escuelas, hoteles, instalaciones deportivas y comercios.

Los dos grandes planes de ensanche para Ámsterdam de Berlage, el Plan Sur (Plan-Zuid) y el Barrio de la Plaza de Mercator (Mercatorplein-buurt), al oeste de la ciudad, también denominado Plan Oeste (Plan West) deben sin duda su fama y reputación a la claridad de la composición urbanística, que se puede atribuir enteramente al madurado talento de Berlage. Pero sin los consecuentes detalles de los edificios y manzanas, la gran calidad de las fachadas y el acabado técnico-constructivo, los acentos y distinciones elegantes y naturales, la rica variedad de tipologías de vivienda, grandes y pequeñas, caras y baratas, y la gran calidad de los acabados del espacio público, puentes, jardines y mobiliario urbano, sin todas estas armoniosas finezas, estas creaciones de Berlage no se habrían convertido en patrimonio histórico-cultural con interés internacional. Diversos arquitectos de la Escuela de Ámsterdam están vinculados al acabado arquitectónico de los planes de ensanche de Berlage, y en especial Michiel de Klerk.

\section{Michiel de Klerk, el arquitecto más significativo de la Escuela de Ámsterdam}

Michiel de Klerk (1884-1923) se hizo famoso de joven gracias a su talento como dibujante, su continua búsqueda de frivolidades y plástica artístico-constructiva, su gran maña con la combinación de urbanismo, edificio, manzana, plano y detalle de fachada, su heroica pericia profesional, con la que creó superficies sorprendentemente bien acabadas. Pero es sobre todo conocido por la impetuosidad y lo polifacético de su increíblemente corta carrera como innovador arquitecto amsterdamés. Hasta 1910 estaba empleado en el despacho de Ed Cuypers (el primo del diseñador de la Estación Central de tren y del Museo Rijks, ambos en Ámsterdam). Entre 1910 y 1915 surgen los primeros diseños relevantes, entonces se desarrolla rápidamente un estilo muy personal, y entre 1915 y 1923 surge una serie de grandes proyectos, sobre todo vivienda social, con los que se convirtió en un polémico pero también uno de los más importantes arquitectos holandeses. De Klerk critica la arquitectura racionalista

\footnotetext{
${ }^{4}$ Nota del traductor: Plan Sur dió lugar al actual distrito Ámsterdam Sur, situado al sur de la ciudad histórica.
} 
de Berlage. En 1916 escribe en un venenoso comentario en el Semanario Arquitectónico (Bouwkundig Weekblad) que él nunca conoció personalmente al señor Berlage, que Berlage quizás había tenido una influencia importante en la profesión "...al haber traído orden en el caos profesional...", pero que Berlage nunca llegó al arte de la arquitectura. "El trabajo de Berlage está demasiado limitado, demasiado exclusivamente técnico y utilitarista, como para poder ser portador alguno de cultura". Aquí se descubre un conflicto generacional entre el por entonces ya reconocido y valorado fundador del nuevo racionalismo de la arquitectura holandesa y un joven y radical diseñador que llevará a remolque a la próxima generación (Bock, 1997, p. 48).

Michiel de Klerk y sus partidarios de la Escuela de Ámsterdam no tuvieron problema alguno para encajarse en la estructura urbanística que Berlage diseñó para Ámsterdam Sur y Ámsterdam Oeste, al oeste de la ciudad histórica, pero al mismo tiempo encontraron allí el espacio para una arquitectura que era más frívola y abundante que la que Berlage tenía en mente en sus famosas perspectivas de vuelo de pájaro de los dos distritos, donde ilustró sus conceptos urbanísticos y también arquitectónicos. Incluso podría ser que justamente la combinación de un plan urbanístico ceñido, esquemático y monumental, junto con la riqueza abundante de monumentalidad arquitectónica y plástica poética hayan dado realmente el carácter a los planes de Berlage. La 'trama' se extiende aquí sobre dos generaciones continuas; el vínculo de unión es la estructura del Plan Sur y el Plan Oeste, que ha resultado ser robusta y sostenible. Fue un arquitecto polifacético, que dejó una obra grande y variada: junto a diversos diseños de concurso muchos grandes complejos de vivienda, viviendas particulares, escuelas, oficinas y sedes bancarias, diseños de interior, muebles y una gran colección de diseños de edificios no realizados.

\section{Principios de la Escuela de Ámsterdam}

Durante los últimos años se está revalorando claramente el trabajo de la Escuela de Ámsterdam como importante corriente dentro de la arquitectura holandesa durante el interbellum. Publicaciones recientes y una gran exposición en el Instituto Holandés de Arquitectura (Nederlands Architectuurinstituut) en Rótterdam son muestras de la gran admiración por el entusiasmo y la originalidad que los trabajos de arquitectos de la Escuela de Ámsterdam irradian. Durante mucho tiempo la situación fue sin embargo bien distinta.

Desde el punto de vista del racionalismo y funcionalismo dominantes desde mediados del siglo XIX hasta los primeros decenios del siglo XX, el 'pintoresco', sugerente, poético, plástico y monumental carácter de la arquitectura de la Escuela de Ámsterdam no fue apenas tomado en serio, mientras que justamente dentro de la corriente principal del modernismo surgían una serie de frivolidades juguetonas como desarrollos desenvueltos de dogmas demasiado estructuralistas y constructivistas.

Los grandes cuatro arquitectos de la Escuela de Ámsterdam, De Klerk, Van der Mey, Kramer y Wijdeveld siguen siendo a pesar de todo emplazados en la tradición de los decimonónicos Jos. Th. Cuypers y Berlage, que son considerados los fundadores de la corriente racionalista de la arquitectura en los Países Bajos, que en el siglo XX obtuvo fama mundial. La Escuela de Ámsterdam no fue ningún accidente ni ninguna corta aberración, sino que encaja en una larga tradición de crítica poética a la frialdad e inaccesibilidad de un racionalismo funcionalista y unidimensional.

Característico de la Escuela de Ámsterdam era que la arquitectura era empleaba sobre todo para dar lugar a una composición urbanística: el conjunto de edificios se desarrolló como una gran composición que operaba con total libertad con respecto de la parcelación y el uso internos. Elementos de esta composición son esquinas distinguidas, líneas de fachadas de composición independiente donde no se reflejan cada vivienda sino una visión arquitectónica 
total de plasticidad y monumentalidad, trabajos de albañilería ricamente detallados, mucha atención por los detalles de bastidores y tejados, y zócalos, entradas y miradores de formas especiales. Aquí se reinventó de nuevo la arquitectura como escultura, como poesía espacial.

Las fuentes que nos quedan de la época parecen señalar que Michiel de Klerk tomó distancia de una forma consciente y explícita del funcionalismo sobrio y rectilíneo. La arquitectura también tenía que ser arte del espacio, desenvuelto y elegante, pintoresco y monumental. Se consideraba que la composición total de las líneas de fachadas era más importante que una expresión 'honrada' de la estructura y parcelario interno.

La arquitectura podía y debía ser una artesanía similar a la escultura, debía, justamente en este punto, aportar más de lo que se podía esperar de los principios racionalistas del oficio de construir.

Michiel de Klerk desarrolló consecuentemente estos principios durante su corta vida en una gran cantidad de edificios y también en estudios de interior y diseños de muebles. Los famosos diseños para viviendas sociales en Ámsterdam eclipsan un gran número de diseños para concursos, pequeños edificios de viviendas y edificios públicos. En todos estos diseños De Klerk se presentaba como artista y profesional virtuoso que gracias a una fantasía inagotable dio forma a una práctica arquitectónica polifacética. Su mentalidad profesional ayudó indudablemente a que diseñadores vinculados a la Escuela de Ámsterdam recibiesen también encargos para el diseño de espacios públicos y mobiliario urbano, puentes, esclusas y pequeñas obras destinadas a instalaciones eléctricas y demás.

\section{Más detalles de algunos proyectos}

El primer projecto en el que De Klerk trabajó como arquitecto independiente, y donde los principios descritos se manifiestan modesta pero inequívocamente, es el Hillehuis, un gran edificio de viviendas con apartamentos de lujo en la esquina de una manzana que da a la plaza Johannes Vermeer (Johannes Vermeerplein), en el distrito del Concertgebouw en ÁmsterdamSur. Diversos elementos de la fachada son en realidad independientes del parcelario interno justamente porque son una respuesta monumental a la estructura urbanística de la trama urbana circundante. Se trata aquí de adornos bastante tradicionales en las fachadas; en proyectos posteriores surgirá a partir de este principio una plástica virtuosa en las superficies de las fachadas con la que manipulará desenvueltamente las formas rigurosas de las manzanas de Berlage. A este edificio le siguió en 1913, por encargo del mismo cliente, esta vez junto al jardín urbano de Spaarndammer (Spaarndammerplantsoen) en Ámsterdam-Oeste, un gran edificio con cajas de escalera que sobresalen en la fachada formando una rítmica sobria pero muy monumental. Después siguieron, desde 1914 a 1921 los internacionalmente famosos edificios situados al otro lado del mencionado jardín urbano de Spaarndammer, realizados por encargo de la corporación de viviendas Eigen Haard. Aquí surge el primer edificio basado completamente en el diseño de De Klerk, y que probablemente es el proyecto de vivienda social en los Países Bajos del que más se ha hablado y escrito. El autor jugó aquí un intrigante juego. Diversas formas fueron agrupadas en distintas escalas dando lugar a una composición harmoniosa, donde la cohesión no surge de repetir elementos de la estructura, sino justamente de conectar entre sí con un movimiento harmonioso distintos acentos dando lugar así a una estructura de movimientos continuos y de gran solidez: el zócalo que contrasta gracias a un trabajo de albañilería pintado con color oscuro, el tejado que sobresale de la línea de fachada con tejas verticales y bordes horizontales sobresalientes que sirven de unión para todos los alféizares de las ventanas. De Klerk no se recató de escandalizar a los racionalistas funcionalistas de su tiempo, o sea, a muchos de sus colegas, con detalles inútiles y señuelos monumentales como una torre de más de 20 metros de altura situado como baliza sobre la cabeza de su edificio en el jardín urbano de Spaarndammer, torre que se ha convertido en un icono impresionante de la vivienda social holandesa. 
En su momento esta sorprendente y particular aberración dentro de la entonces dominante arquitectura Berlagiana provocó indudablemente mucha polémica en el mundo profesional. El prominente arquitecto Jos. Cuypers, hijo del autor de la Estación Central y el Rijksmuseum en Ámsterdam, dimitió como miembro de la Comisión del Buen Aspecto ${ }^{5}$ porque no podía aceptar que la mayoría de sus miembros aceptase lo que él denominó la 'arquitectura de pegatinas' (plakarchitectuur) de De Klerk. Otro arquitecto, y crítico, Jan Gratama, opinaba que "...los detalles no sólo (son) sospechosos, sino también rebuscados, extraños e insanos (...) Así por ejemplo un balcón que sobresale ladeado, puertas enmarcadas con líneas ladeadas, trabajos de albañilería sin sentido, una coordinación rara de ventanas con la que sobresale una viga de la fachada en el último piso y que sirve con la ayuda de una polea subir y bajar el mobiliario durante las mudanzas, todo muy raro..." (V. Stissie en Bock, M., 1997, p. 57).

Pero, indudablemente gracias al apoyo de Wibaut y Keppler, Michiel de Klerk pudo en los últimos cinco años de su vida realizar aún unos cuantos grandes proyectos de edificios de vivienda, proyectos con los que marcó el tono del desarrollo posterior de los grandes planes de ensanche de Berlage en Amsterdam hasta finales de los años '30. La Escuela de Ámsterdam se convirtió en una escuela con seguidores en Ámsterdam y también en el resto de los Países Bajos. Uno detrás de otro se ejecutaron proyectos de Michiel de Klerk: P.L. Takbuurt, la Plaza de Ronner (Ronnerplein), y el vecindario detrás de la avenida de Vrijheid. Otros arquitectos como Greiner, Rutgers, Van Epen, Wijdeveld, Kropholler, Staal y Boetrenbrood siguieron su camino (ver Casciato, M., 1991, p. 120 y siguientes).

\section{La apreciada herencia: política de restauración en los años noventa}

El gobierno holandés ha desarrollado desde los años setenta una política de estimulación de envergadura destinada a la mejora de barrios y viviendas deteriorados, a la gestión y reestructuración de distritos residenciales y a un tratamiento cuidadoso de monumentos con indudables valores culturales e históricos. Esta política se apoyaba en el mayor aprecio que tanto la sociedad como la política locales profesaban y profesan por la herencia arquitectónica y urbanístico-arquitectónica de Ámsterdam.

Pero inicialmente también se cometieron errores. Cuando a comienzos de los años setenta se inició con las grades operaciones de mejora y rehabilitación de viviendas no todos los empleados de las corporaciones de viviendas, ni todos los funcionarios y políticos del Ayuntamiento, eran conscientes del significado cultural e histórico de las viviendas que tenían entre manos. En ocasiones de destruyeron detalles de fachada sin que fuera necesario, se aplanaron los detalles en madera de ventanas y bastidores con materiales sintéticos de fácil mantenimiento, miradores y tejados fueron cubiertos con planchas de materiales baratos, valiosos detalles de albañilería desaparecieron detrás de nuevas fachadas modernas, puertas de madera muy elaboradas dieron paso a puertas planas, en los trabajos de pintura no se prestó atención a los matices de los colores originales, detalles especiales como la numeración de las viviendas y los buzones del correo fueron 'modernizados'. Los resultados de estas operaciones de mejora fueron peores en las viviendas particulares, generalmente dispersas, que en los grandes paquetes de viviendas propiedad de las corporaciones, ya que en las primeras la renovación se acometió a menudo edificio a edificio, lo que llevó a una sobreposición de lo viejo y lo nuevo, y a menudo también a un tratamiento distinto de detalles iguales en un mismo conjunto. Muchas de estas intervenciones no requerían de una licencia de obras, de modo que muchas de ellas no fueron controladas por las comisiones del buen aspecto o por los especialistas en monumentos del Ayuntamiento. En un primer momento las

\footnotetext{
${ }^{5}$ Nota del traductor La Welstandscommissie o Schoonheidscommissie [Comisión del Buen Aspecto], formada generalmente por arquitectos y que vela por la calidad estética de las ciudades y pueblos, tenía y tiene aún hoy un papel decisivo en el proceso de tramitación de las licencias de edificación.
} 
críticas surgieron en el seno de reducidos círculos de arquitectos, historiadores del arte e inquietos vecinos (Gaillard y Dokter, 1992).

A partir de finales de los años ochenta el tema de la 'renovación cuidadosa' se convirtió en un tema de reconocido interés dentro de la política local. Se creó un fondo de subvenciones adicional para aquellos que cumpliesen determinadas condiciones, se creó la figura de un supervisor que asesoraba a los propietarios de vivienda y los vecinos, y las comisiones del buen aspecto acentuaron su control, también para aquellas intervenciones de menor envergadura en las fachadas. El trabajo del supervisor, del departamento municipal de Control de la Construcción y la Vivienda y de la Comisión Amsterdamesa del Buen Aspecto se plasmó en una importante serie de publicaciones que inventarizaban y normalizaban, y donde se podían encontrar instrucciones para el detallamiento técnico deseado para un gran número de operaciones de restauración (ver Luursema y Mulder, 1995; y Bolhuis et alii, 2000).

Existe también un cuerpo importante de conocimiento sobre el valor de la herencia construida, conocimiento en el que en los últimos años se ha profundizado y que ha sido difundido mediante investigación, publicaciones y exposiciones. Gracias a ello la herencia de los años veinte y treinta ha sido modernizada en profundidad en los últimos años, al mismo tiempo que restaurada conforme a las ambiciones originales. 


\section{Bibliografía}

Bock, M., "Michel de Klerk; bouwmeester en tekenaar van de Amsterdamse School 18841923" (Michel de Klerk: maestro de la construcción y dibujante de la Escuela de Ámsterdam 1884-1923), Rotterdam, Nai, 1997.

Borrie, G., "F.M. Wibaut, mens en magistraat" (F.M. Wibaut, ser humano y magistrado), versión revisada, Sdu, Den Haag, 1987.

Bolhuis, G. (red), y Stedelijke Woningdienst Amsterdam (Departamento de la Vivienda de Ámsterdam), “Atlas Gordel 20-40" (Atlas Cinturón 20-40), Amsterdam, De Balie, 2000.

Casciato, M., "De Amsterdamse School" (La Escuela de Ámsterdam), Rotterdam, Editorial 010, 1991.

Gaillard, K. y Dokter, B., "Berlage en Amsterdam Zuid" (Berlage y Ámsterdam-Sur), Rotterdam, Editorial 010, 1992.

Mulder, B y E. Luursema, "Handboek renovatie gordel 20-40; architectuurherstel in Amsterdam" (Manual restauración cinturón 20-40: rehabilitación de arquitectura en Ámsterdam), Bussum, Thoth, 1995.

Ottens, G., "Ik moet naar een kleinere woning onzien, want mijn gezin wordt te groot" (Tengo que buscar una vivienda más pequeña, ya que mi familia se hace demasiado grande), Amsterdam, Gemeentelijke Dienst Volkshuisvesting (Departamento Municipal de la Vivienda Social), 1975.

Ouwehand, A. \& G. van Daalen, "Dutch housing associations, a model for social housing" (Las asociacions de vivienda holandesas, un modelo para la vivienda social), DUP Satelite, Delft 2002.

Pistor, R. y Polak, B. "Nooit voltooid, de ruimtelijke ordening in Amsterdam" (Nunca acabada, el urbanismo en Ámsterdam), Amsterdam, Dienst Ruimtelijke Ordening Amsterdam (Departamento Municipal de Urbanismo de Ámsterdam), 1994. Existe una edición en inglés de esta obra.

Polano, S. y Fanelli, G., "Hendrik Petrus Berlage, het complete werk" (H.P. Berlage, la obra completa), Alphen aan den Rijn, Atrium,1988.

Roegholt, Richter. "Amsterdam na 1900" (Ámsterdam después de 1900), Den Haag, Editorial Sdu, 1993.

Schaar, J. van der, "Groei en bloei van het Nederlandse volkshuisvestingsbeleid" (Crecimiento y florecimiento de la Política Neerlandesa de Vivienda Social), Delftse Universitaire Pers, Delft 1987.

Slot, E., Grond, "Het schaarse erfgoed; 75 jaar Grondbedrijf Amsterdam 1921-1996" (La escasa herencia: 75 años de la Empresa del Suelo de Ámsterdam), Amsterdam, Gemeentelijk Grondbedrijf Amsterdam (Empresa Municipal del Suelo de Ámsterdam), 1996.

Smit, F., "Arie Keppler, woninghervormer in hart en nieren" (Arie Keppler, reformador de la vivienda en corazón y riñones), Bussum, THOTH, 2001. 